\title{
Learning Stochastic Adaptive Control using a Bio-Inspired Experience Replay
}

This paper was downloaded from TechRxiv (https://www.techrxiv.org).

LICENSE

CC BY 4.0

SUBMISSION DATE / POSTED DATE

03-03-2022 / 08-03-2022

CITATION

CHAFFRE, Thomas; SANTOS, paulo; LE CHENADEC, Gilles; CHAUVEAU, Estelle; CLEMENT, Benoit; Sammut, Karl (2022): Learning Stochastic Adaptive Control using a Bio-Inspired Experience Replay. TechRxiv. Preprint. https://doi.org/10.36227/techrxiv.19297577.v1

$\mathrm{DOI}$

10.36227/techrxiv.19297577.v1 


\title{
Learning Stochastic Adaptive Control using a Bio-Inspired Experience Replay
}

\author{
Thomas Chaffre, Paulo E. Santos, Gilles Le Chenadec, \\ Estelle Chauveau, Karl Sammut, Senior Member, IEEE, Benoit Clement
}

\begin{abstract}
Deep Reinforcement Learning (DRL) methods are dominating the field of adaptive control where they are used to adapt the controller response to disturbances. Nevertheless, the usage of these methods on physical platforms is still limited due to their data inefficiency and the performance drop when facing unseen process variations. This is particularly perceived in the Autonomous Underwater Vehicles (AUVs) context as studied here, where the process observability is limited. To be effective, DRL-based AUV control systems require the use of methods that are data-efficient (in order to reach a satisfactory behavior with a sufficiently fast response time) and are resilient (to ensure robustness to severe changes in operating conditions). With this ambition, we study in this paper the effect of the Experience Replay (ER) mechanism on the performance variation of a DRL-based stochastic adaptive controller. We propose a new ER method (denoted as BIER) that takes inspiration from the biological Replay Mechanism and compare it to the standard method denoted as CER. We apply it to the Soft Actor-Critic, a maximum entropy DRL algorithm, for use with an AUV maneuvering task that consists in stabilizing the vehicle at a given velocity and pose. The training results show that our BIER method exceeds the performance of the nonadaptive optimal model-based counterpart of the controller in less than half the number of episodes compared to CER. We proposed different evaluation scenarios of increasing complexity as measured by desired velocity value and amplitude of current disturbance. Our results suggest that the BIER method achieves improved learning stability and better generalization abilities.
\end{abstract}

Index Terms-Deep reinforcement learning (DRL), neural networks, machine learning (ML), adaptive control.

\section{INTRODUCTION}

\section{A. Adaptive Control}

Autopilots for unmanned systems are usually designed based on the feedback provided from velocity and orientation sensors. In the case of autopilot systems for autonomous underwater vehicles (AUVs), the main objective in the design is to compensate for waves and current-induced disturbing forces acting on their body. Existing AUV autopilots are however only able to compensate for low-frequency components of seainduced disturbances. It seems natural to assume that the AUV performance could be improved by taking the nature of the disturbances into account in the design of the autopilot.

Adaptive control [1] provides what seems to be an ideal framework to this end. The objective of this technique is

This work was supported by ISblue project, Interdisciplinary graduate school for the blue planet (ANR-17-EURE-0015) and co-funded by a grant from the French government under the program "Investissements d'Avenir".

The first author's scholarship is jointly supported by the doctoral school of Bretagne-Loire University with a grant from the Brittany Region under the program "Mobilité Internationale" and by Flinders University with a grant provided by the South Australian Government. to adjust automatically the control parameters when facing unknown or time-varying processes such that the desired performance threshold is met. Developed in the late 1950s, adaptive control frameworks have been considerably expanded and used in various fields, their application has been facilitated by the rapid progress in microelectronics and the increasing interaction between laboratories and companies, from aerospace to maritime industries. As a result, adaptive controllers started to be widely adopted in the industry in the early 1980s. It was established at that time that robust designs with fixed parameters are too limited to handle complex regimes. The study of adaptive controllers for AUV manoeuvring is associated with various challenges, including:

Unknown dynamics: the uncertainty associated with describing precisely the states of waves or currents is high. This, together with its dynamic nature, prevents linear feedback control methods from achieving optimal performance of the plant. This becomes more critical in the presence of changes in weather conditions that impose a multiplicative factor in the component of the induced forces. The disturbance period will also vary with the speed of the vehicle and its orientation relative to the waves.

Nonlinearity: the controller response at some operating points must be overly conservative in order to satisfy the specification at other operating points. This is difficult to achieve for fixed parameters obtained through local linearization, that do not encompass the entire regime envelope.

Thruster efficiency: a fully-actuated vehicle can often become underactuated as its forward speed increases. This is especially true for hovering AUVs which are designed to move at low speeds and steer using their thrusters only. As the forward speed increases, the efficiency of lateral motions is drastically reduced, making it impossible for the platform to account for pure lateral motions.

System reliability: if the performances of one or more thrusters become increasingly less effective, the control system should be able to detect this and engage a new control algorithm specially designed to accommodate the failures and, if possible, to complete the mission.

A class of adaptive control methods, known as learningbased adaptive controllers, have been developed to tackle some of these limitations. This family of solutions uses machine learning algorithms capable of compensating for the unknown part of a process while also maintaining optimal control of its known part using traditional methods. 


\section{B. Learning-based adaptive control}

Real-world systems are in general nonlinear and their motion equations, parameters and system measurements are affected by uncertainty. A realistic scheme is to consider that the process model is partially available. In learningbased adaptive controllers, model-free algorithms are used to mitigate this lack of a complete description of the process by finding (learning) an approximate representation of the unknown parts, or by fitting (tuning) the best control parameters for a target behavior. The dynamics of such a system can be represented as the sum of known $\left(f_{1}\right)$ and unknown $\left(f_{2}\right)$ parts:

$$
\begin{aligned}
& \dot{x}(t)=f_{1}(t, x, u)+f_{2}(t, x, p), \\
& y(t)=h(t, x, u),
\end{aligned}
$$

where classical model-based control methods can be used to efficiently control $f_{1}$, and $f_{2}$ which can be approximated by model-free learning algorithms. In other words, learningbased control methods take advantage of the fast convergence and robustness to uncertainty of learning algorithms to approximate an unknown performance function, while applying model-based control laws to maintain some stability requirements of the system. More recently, in Neural-Network (NN) learning-based control design, the unknown part of the model can be estimated by a NN, whose weights are obtained using some model-free optimization procedure. Among the various techniques, a prominent candidate to that end is Deep Reinforcement Learning (DRL).

In DRL, the learning process happens through the interaction between an agent and the environment [2]. DRL is traditionally defined as a Markov Decision Process (MDP) expressed as the tuple $\langle S, A, T, R\rangle$, in which:

$S$ is the set of possible states;

$A$ is the set of actions that can be executed by the agent;

$T$ is the transition function that defines the probability of reaching a successor state $s^{\prime} \in S$, from the application of an action $a \in A$ in a state $s \in S$;

$R$ is the reward function.

The DRL general framework can be summarised as follows:

1) At an instant $t$, an action $a \in A$ is chosen by the agent in a state $s \in S$;

2) The execution of this action leads the agent to a state $s_{t+1} \in S$ and the agent receives a scalar value $r_{t}$, the reward signal. This signal is a numerical representation of the action outcomes with respect to a reward function $R(s)$. The goal of DRL is to maximize this reward function;

3) Finally, the agent updates the value of executing action $a$ based on the received reward, according to the specific DRL algorithm applied.

In the domain of optimal control, the agent is identified with the controller, environment is the controlled system (or plant) and action is the control signal [2].

Among the various existing DRL algorithms [3], Deep Policy Gradient methods (that use gradient descent for the purpose of optimizing a decision making function, denoted as policy, with respect to the expected return) are deemed the most suitable method for handling robotic domains for the following reasons:

1) Domain dimensionality: the large size of the state-space in a real robot environment makes the application of value iteration based methods unfeasible;

2) Non-observable disturbances: any real environment, beyond toy scenarios, is partially observable. In this context, environment modelling and trajectory planning are extremely difficult tasks for any model-based or value-based DRL method, since unexpected states can corrupt the remaining part of the estimated trajectory, resulting in failures.

Deep Policy Gradient methods are essentially relying on the Actor-Critic architecture [4], where a value and a policy function are estimated simultaneously in order to improve the agent performance. This formulation has led to the development of what is known as off-policy methods where the estimates of these functions can be improved using the Experience Replay (ER) mechanism. ER is the state-of-the-art method for the automatic selection of past experience to improve an agent's future behavior, allowing the use of samples from various distribution. In contrast, with the on-policy formulation, only samples generated by the same policy can be considered for the optimization process.

The off-policy Deep Policy Gradient methods are increasingly being applied to AUVs adaptive control [5]-[8] where the standard ER method is used to improve DRL-based agents for the purpose of adapting to process variations. These works rely on deterministic policies that are highly stable during training but display lower adaptation abilities compared to their stochastic counterparts. Nevertheless, the performance of Deep Policy Gradient methods is highly sensitive to the distribution shift problem that is in the context of DRL, the difference between the training and evaluation set of states. A key element toward the reduction of this sensibility is the ER mechanism, and its effect on the resulting policy is the main focus of this paper.

This paper is organized as follows: in Section $\Pi$ the ER mechanism is introduced with its standard formulation in DRL and its biological counterpart. In Section III we present an application of learning-based adaptive control theory for the control of an AUV. The detailed architecture of the learningbased adaptation is provided in Section III-B We present our proposed ER approach in Section IV] The training settings are provided in Section $\mathrm{V}$ along with the training results. The evaluation scenarios are described in Section VI with an analysis of the results, leading to some open questions and perspectives for future work.

\section{EXPERIENCE REPLAY}

The concept of ER [9] employs the agent's past experience to improve its current behavior. It aims to artificially make the agent's experience look Independent and Identically Distributed (IID). This is highly desirable in order to not concentrate the updates to a limited area of the desired functions.

Given that an agent's experience at the time step $t$ is defined as the quadruplet $e_{t}=\left(s_{t}, a_{t}, r_{t}, s_{t+1}\right)$, the ER method 
consists of storing (at each time step) the experience $e_{t}$ in a memory unit $\mathcal{D}=\left\{e_{1}, \ldots, e_{t}\right\}$ of fixed size, also known as the replay buffer. Then, the neural networks are trained by performing mini-batch gradient descent of past experiences randomly pooled over the replay buffer. The estimators are hence trained on IID samples that are generated by various trajectories and policies and they are, therefore, more representative of the true function. However, even with this first ER formulation, there is a great number of parameters that are usually ignored despite having an impact on the learning performance, including:

The replay buffer size: the total number of transitions that the replay buffer can store. When its maximum size is reached, the replay buffer is accessed in a first-in-first-out fashion. The bigger the replay buffer size, the more the data will look like it is IID, which in turn improves the gradient update quality. However, if the replay buffer is too big, an important transition will have much less chance of being used to update the policy, which could impair the learning process. In contrast, if the replay buffer is too small, the learned policy can be the result of an overfitting process on recent transitions, which precludes performance improvement.

The age of a transition: the number of gradient steps taken by the agent since the transition was generated. This value can be seen as a measure of the extent to which the transitions stored in the Replay Buffer are off-policy, as it tells us how different the current policies are from those stored in the buffer. The age of the oldest policy stored increases with respect to the buffer size.

The replay ratio: the number of gradient updates per environment transition. It can be viewed as a measure of the frequency at which the agent is learning using existing data versus learning from collecting new experiences.

The size of the replay buffers, however, can impact negatively on the learning performance [10]. There are two competing methods that can be used to solve this issue: the Combined ER (CER) [10] and the Prioritized ER (PER) [11]. CER consists of adding the latest transition performed to the mini-batch pooled over the replay buffer, whereas with PER important transitions, as measured by their associated TDerror 22, 23, are given a higher probability to be used in the gradient updates. Using CER, however, the last transition will undoubtedly be sampled and instantly affect the policy.

Nevertheless, even with CER, a drop in performance was observed for certain sizes of replay buffer, at some point of the training (even when tuning the learning rate). This behavior was related to the process itself rather than to the aforementioned parameters [10]. As written in [10] "CER is a workaround ... and future effort should focus on developing a new principled algorithm to fully replace ER.” In this paper, we propose a new ER mechanism with the ambition to decouple the performance of the agent from process complexity (as observed with CER).

A recent detailed analysis of ER was provided in [12], where an analysis of the effects of the aforementioned parameters was presented. Several conclusions on how the parameters can affect the learning dynamics were drawn, which motivated the ER design proposed in this work. These conclusions can be summarised as follows:

- Increasing the replay capacity while fixing the age of the oldest policy improves the performance because it lowers the chances of overfitting to a small subset of (state,actions).

- As the agent trains, it spends more time in higher quality regions of the environment (as measured by rewards), thus learning to better estimate the return in such regions leads to further gains in performance.

- Increasing the buffer size with a fixed replay ratio has varying improvements. The replay ratio stays constant when the buffer size is increased because of both the replay capacity and the age of the oldest policy increase. If one of these two factors is independently modulated, the replay ratio will change.

The design of the proposed approach for ER has been mostly motivated by these findings which we tried to incorporate in the ER scheme. A recent study [13] undertook a comprehensive comparison between the replay mechanism that takes place in biological brains and those in artificial learning systems. We list below some findings related to DRL only.

Replay (in biological systems) is temporally structured. Temporally correlated experience sequences are used for learning and memory combination. This allows for more combinations of neurons which leads to faster emergence of temporal waking experiences. This feature is largely ignored by existing methods that only replay static, uncorrelated inputs.

Replay is modulated by reward and only a few selected experiences are replayed. It seems intuitive that not all experiences are useful for learning a new task. Some experiences are more important than others because they incorporate higher quality information about the process dynamics. The challenge here is twofold: how to model this information quality and how to measure it.

Replay is treated differently for novel versus non-novel inputs. This allows for selective replay to be weighted by novelty. Biological systems tend to reduce drastically the attention given to old experiences versus that given to recent ones as the latter contain more information within them.

In this paper, we propose a new ER mechanism to include these biological insights while keeping in mind the constraints related to the regression problem.

\section{A LEARNING-BASED ADAPTIVE CONTROLLER}

In this paper, we address the control problem of AUV manoeuvring, which can be summarized as the stabilization of an underwater vehicle at a fixed velocity and orientation ( $x_{r e f}$, with $\dot{x}_{r e f}=0$ ). The state vector is hence defined as $x=\left[\begin{array}{llllll}x & y & z & \phi & \theta & \psi\end{array}\right]^{T}$. The vehicle is fully actuated but subject to external disturbances (sea currents) that are here not observable (noted that relative current can be estimated with some types of DVL incorporating a bottom lock). As defined in Section $\mathrm{I}-\mathrm{B}$, the process dynamics can be framed as the combination of its known $f_{1}$ and unknown $f_{2}$ parts. Our approach consists in using the knowledge of $f_{1}$ to design a model-based control structure, which is then combined 
with a model-free learning algorithm to compensate for $f_{2}$. The resulting learning-based controller guides the controller parameters toward the optimal ones that best compensate for both $f_{1}$ and $f_{2}$ without prior estimation of some process parameters, leading to a Direct Adaptive Control scheme. Let the error between the present $\left(\bar{x}_{i}\right)$ and the desired $\left(x_{r e f_{i}}\right)$ state variable be defined as $e_{i}=x_{r e f_{i}}-\bar{x}_{i}$.

The task of steering the AUV outputs in order to maintain the error signals within a specific threshold, over a predefined amount of time (guaranteeing the vehicle stabilization), can be achieved if the following control objective is met:

$$
\forall i \in \mathbb{R}^{u}, \forall t^{\prime} \in[t-\varsigma, t], \exists !\left|e_{i}\left(t^{\prime}\right)\right|>\chi
$$

where $\mathbb{R}^{u}$ is the space of control inputs, the current time step is denoted as $t$ and $\varsigma$ is the length of the period of time over which we want all the errors $e_{i}$ to be less than the desired value $\chi$. This class of control objective is used in various AUV missions, such as autonomous docking or underwater inspection, where a conservative regulation of the vehicle's outputs is required.

We use the ROS-based UUV Simulator [14] which contains a small library of different vehicles of cubic and torpedo shapes. For this study, we used the RexROV2 platform, a cubic shape AUV1 The UUV Simulator can simulate several current and wave disturbances, thruster dynamics, and body wrench disturbances. When incorporated in simulations, the induced forces have a realistic physical impact on the robot and fluid dynamics. The sea current disturbance (which is the main focus of this study) is modeled as a uniform force acting over the Gazebo environment. This force is represented by a linear velocity, $v_{c}$ (in $m . s^{-1}$ ), a horizontal $h_{c}$ and a vertical angle $j_{c}$ (measured in radians). The simulated RexROV2 platform is equipped with an IMU which feedbacks its linear velocities and orientation (Euler angles). These variables are accessible through ROS topics, which are essentially data pipelines to access the state of the simulated objects. Our software architecture consists in using the simulation metadata to train the learning algorithms considered in this work.

\section{A. Design of the model-based part of the controller}

We introduce now a simple learning-based adaptive controller formulation. We first proposed it in [15] where we demonstrated its superiority to its nonadaptive optimal modelbased counterpart (denoted in this manuscript as OFP for Optimal Fixed Poles controller) that we used here to compare our controllers. In particular, we show in [15] that only the learning-based adaptive controller (that we introduce now) was able to regulate the vehicle against time-varying disturbances. Moreover, we provide in this paper an enhanced model-based design that leads to better performance. This work assumes that the controlled vehicle is fully observable and controllable. This means that each of the vehicle's DoF is measurable and the desired vehicle states (within the operating regimes) are supposed to be accessible. In this context, a PID controller is a suitable method to regulate the process. We can take into

\footnotetext{
${ }^{1}$ See our last study $[15]$ for further information on the simulated vehicle.
}

account the current disturbance by considering the steady-state error variable $\sigma=\int_{0}^{t} e(\tau) d \tau$. We can rewrite the state-space equations with the augmented state vector $X=[\sigma, e, \dot{x}]$ as:

$$
\frac{d}{d t}\left[\begin{array}{c}
\sigma \\
e \\
\dot{x}
\end{array}\right]=\underbrace{\left[\begin{array}{ccc}
0 & 1 & 0 \\
0 & 0 & 1 \\
0 & 0 & 0
\end{array}\right]}_{\mathrm{A}}\left[\begin{array}{c}
\sigma \\
e \\
\dot{x}
\end{array}\right]+\underbrace{\left[\begin{array}{l}
0 \\
0 \\
1
\end{array}\right]}_{\mathrm{B}} u .
$$

The PID state-space representation is given by:

$$
\dot{X}=(A-B K) X \text {. }
$$

The PID control law can then be derived as:

$$
u=k_{p} e+k_{i} \sigma+k_{d} \dot{x},
$$

with $k_{p}, k_{i}$ and $k_{d} \in \mathbb{R}^{+}$. Among the various procedures and rules that can be applied to tune the PID gains [16], a fundamental technique consists of assigning a set of specific values, $P=\left\{\lambda_{1} \lambda_{2} \ldots \lambda_{n}\right\}$, to the eigenvalues of the feedback loop $A-B K$. Given that these eigenvalues determine the poles of all the transmittances where the associated state matrices are involved, this procedure is denoted as Pole-Placement. We can define a (normalized) control polynomial as

$$
C(s)=s^{n}+c_{1} s^{n-1}+\cdots+c_{n-1} s+c_{n},
$$

whose roots are the $\lambda_{i}$, which can be assigned the characteristic polynomial of $A-B K$ with:

$$
C(s)=\operatorname{det}(s \mathbf{I}-(A-B K)) .
$$

Equations (4) and (7) yield

$$
\begin{aligned}
|A-B K-\lambda \mathbf{I}| & =-\lambda\left(\lambda\left(k_{d}+\lambda\right)+k_{p}\right)-k_{i}, \\
& =-\lambda^{3}-\lambda^{2} k_{d}-\lambda k_{p}-k_{i}, \\
& =0 .
\end{aligned}
$$

The desired $\lambda_{i}$ are solutions of

$$
\lambda^{3}+\lambda^{2} k_{d}+\lambda k_{p}+k_{i}=0 .
$$

To ensure the control loop stability, the poles of (9) must be placed in the complex left half-plane (i.e. $A-B K$ is Hurwitz). For this purpose, the poles of the controller (5) must be solutions to 9 . In order to derive such poles $\left(\tau_{1}, \tau_{2}, \tau_{3}\right) \in \mathbb{R}^{+}$, we the following eigenvalues design is considered:

$$
\lambda_{1}=\frac{-1}{\tau_{1}} ; \lambda_{2}=\frac{-1}{\tau_{2}} ; \lambda_{3}=\frac{-1}{\tau_{3}}
$$

The Pole-Placement design can be written as:

$$
\left\{\begin{array}{l}
\frac{-1}{\tau_{1}^{3}}+\frac{k_{d}}{\tau_{1}^{2}}-\frac{k_{p}}{\tau_{1}}+k_{i}=0 \\
\frac{-1}{\tau_{2}^{3}}+\frac{k_{d}}{\tau_{2}^{2}}-\frac{k_{p}}{\tau_{2}}+k_{i}=0 \\
\frac{-1}{\tau_{3}^{3}}+\frac{k_{d}}{\tau_{3}^{2}}-\frac{k_{p}}{\tau_{3}}+k_{i}=0
\end{array}\right.
$$

Since $\tau_{1}, \tau_{2}$ and $\tau_{3}$ are solutions to 9 , it follows that:

$$
\left[\begin{array}{ccc}
1 & \frac{-1}{\tau_{1}} & \frac{1}{\tau_{1}^{2}} \\
1 & \frac{-1}{\tau_{2}} & \frac{1}{\tau_{2}^{2}} \\
1 & \frac{-1}{\tau_{3}} & \frac{1}{\tau_{3}^{2}}
\end{array}\right]\left[\begin{array}{c}
k_{i} \\
k_{p} \\
k_{d}
\end{array}\right]=\left[\begin{array}{c}
\frac{1}{\tau_{1}^{3}} \\
\frac{1}{\tau_{2}^{3}} \\
\frac{1}{\tau_{3}^{3}}
\end{array}\right] \Leftrightarrow M K^{T}=N
$$


The resulting gains of the control law (5) are obtained by transforming back the poles with $K^{T}=M^{-1} N$ as:

$$
k_{i}=\frac{1}{\tau_{1} \tau_{2} \tau_{3}} ; k_{p}=\frac{\tau_{1}+\tau_{2}+\tau_{3}}{\tau_{1} \tau_{2} \tau_{3}} ; k_{d}=\frac{\tau_{1} \tau_{2}+\tau_{1} \tau_{3}+\tau_{2} \tau_{3}}{\tau_{1} \tau_{2} \tau_{3}}
$$

With the mapping in Eq. (13), the bounds for the controller parameters can be defined based on control constraints that are easier to derive in the poles domain. In the present case, with the design of Eq. (10), for any $\tau_{i}>0$, the poles of the feedback loop are placed on the x-axis of the complex left halfplane. By doing that, we set the desired oscillation frequency and percentage overshoot to 0 . This leaves us with the settling time requirement to define in order to set the desired distance from the $y$-axis and thus bound the value of the space of poles. In accordance with the control objective shown in Eq. (2), we define the desired maximum settling time of the closed loop control $\varsigma=10$ seconds as the maximum time after which we want the system outputs to stay around $\chi=5 \%$ of its desired values. The upper bound of the space of poles is derived as:

$$
\lambda_{\max }=\frac{\ln (\chi)}{\varsigma}=\frac{\ln (0.05)}{10}=-0.3 .
$$

Therefore $\lambda_{i} \leq \lambda_{\max }$ (Eq. (10), from which we can derive the upper bound of the poles

$$
\tau_{\min }<\tau_{i} \leq \frac{1}{-\lambda_{\max }}=3.333=\tau_{\max }
$$

We set $\tau_{\min }=0.025$ because, for lower values, the control inputs are too expensive in terms of control efforts and too aggressive for our control objective. Thus, the bounds of the poles are defined as:

$$
0.025 \leq \tau_{i} \leq 3.333
$$

There is a solution for all $C(s)$ in Eq. (6) if and only if the pair $(\mathrm{A}, \mathrm{B})$ is controllable, that is assumed here. In the case of a Single-Input system, the solution is unique. In the case of Multi-Input systems, as studied here, the number of free components of the matrix $K$ is greater than the $n$ eigenvalue constraints. Accordingly, there exist multiple solutions, among which it is not trivial to define an optimal one.

When no model of the uncertainties is provided, as investigated here, model-free adaptation can be exploited. In order to take into account the uncertainties in the poles selection, we apply DRL to build a stochastic predictive model $\pi_{\mu}$ that maps a state vector $s_{t}$ into the pole values:

$$
\begin{aligned}
& \pi_{\mu}: s_{t} \mapsto\left[\tau_{v x} ; \tau_{v y} ; \tau_{v z} ; \tau_{\phi} ; \tau_{\theta} ; \tau_{\psi}\right], \\
& \pi_{\mu}: s_{t} \mapsto \mathcal{N}\left(\tau_{i}\right),
\end{aligned}
$$

where $\operatorname{dim}(\tau)=18$ and $\mathcal{N}\left(\tau_{i}\right)$ is the probability density function of $\tau_{i}$ that is modeled by a Normal distribution as:

$$
\mathcal{N}\left(\tau_{i}\right)=\left(2 \pi \mu_{i}\right)^{-1 / 2} \exp \left\{-\frac{1}{2 \mu_{i}}\left(x-\lambda_{i}\right)^{2}\right\},
$$

where $\lambda_{i} \in \mathbb{R}$ and $\mu_{i} \in \mathbb{R}^{+}$are the mean and variance of $p\left(\tau_{i}\right)$ that are estimated by the Policy network. Therefore, the outputs of the Policy network are the 18 pairs of $(\lambda, \mu)$ representing the Normal distributions $\mathcal{N}\left(\tau_{i}\right)$ used to sample the poles for each degree of freedom. Designing this stochastic function (17) is numerically expensive due to the dimensions of the underlying spaces, excluding real-time computation with model-based methods only. The DRL framework allows us to iteratively build an estimate of this optimal mapping function. We compare the resulting DRL-based controllers to the OFP controller (provided by the UUV Simulator) whose pole values have been optimized using SMAC [17], a modelbased method, thus without learning. The OFP controller is not adaptive as the poles are fixed. Details on the resulting poles can be found on the UUV Simulator website We present in the next section our use of DRL to adapt the pole values.

\section{B. Design of the DRL-based model-free learning procedure}

The related methods cited earlier in Section $\mathbb{I}$ are mostly relying on the DDPG and TD3 algorithms. They are known to involve intensive tuning of hyperparameters to work properly. Additionally, in order to take into account the ocean current disturbances, one designer might favor a stochastic policy that is known to be more robust to uncertainties and to partially observable processes (at the cost of being less stable during training). For these reasons, we chose to use another Deep Policy Gradient method, named Soft Actor-Critic. It has been exploited to solve many DRL benchmark environment using the exact same NN architecture and hyperparameters. A complete description of this algorithm is detailed next. Our learning-based architecture is therefore composed of 3 main items that we present now.

1) Soft Actor-Critic: The Soft Actor-Critic (SAC) [18][20] is a current state-of-the-art Policy Gradient algorithm integrating three key components:

- An improved exploration and stability in performance thanks to entropy maximization [21].

- An Actor-Critic architecture [4] with separate Values and Policy networks.

- An off-policy formulation enabling the use of past collected data with Experience Replay [9].

Instead of optimizing only the expected sum of rewards, the objective function of SAC also maximizes the entropy of the behavior policy, that is weighted by a constant, $\alpha$, as follows:

$$
J_{S A C}\left(\pi_{\mu}\right)=\sum_{t=1}^{T} \mathbb{E}_{\left(s_{t}, a_{t}\right) \sim \rho_{\pi_{\mu}}}\left[r\left(s_{t}, a_{t}\right)+\alpha H\left(\pi_{\mu}\left(. \mid s_{t}\right)\right)\right],
$$

where

$$
H\left(\pi_{\mu}(. \mid s)\right)=-\sum_{a \in A} \pi_{\mu}(a \mid s) \log \pi_{\mu}(a \mid s)
$$

is known as the Shannon entropy measure of the policy $\pi$ that is represented by an ANN parameterized by $\mu$. By trying to maximize the entropy of the policy and the reward at the same time, the policy is driven to take the best actions while remaining as random as possible. This results in better exploration and improved robustness to uncertainty thanks to entropy maximization [21], [22].

2 https://uuvsimulator.github.io 
For this purpose, the entropy term is explicitly incorporated in the State-Value function $V\left(s_{t}\right)$ as:

$$
\begin{aligned}
V\left(s_{t}\right) & =\mathbb{E}\left[Q\left(s_{t}, a_{t}\right)+\alpha \mathcal{H}\left(\pi_{\mu}\left(. \mid s_{t}\right)\right)\right], \\
& =\mathbb{E}\left[Q\left(s_{t}, a_{t}\right)-\alpha \log \pi_{\mu}\left(a_{t} \mid s_{t}\right)\right],
\end{aligned}
$$

where $\alpha$ is controlled indirectly by the reward scale (see Section V-B. In order to reduce Actor-Critic value overestimation [23], [24], the State-Value function is estimated by an ANN parameterized by $\Psi$ using the minimum of two different Q-Value estimates represented by two ANNs parameterized by $\Upsilon_{1}$ and $\Upsilon_{2}$, respectively. We used TD-Learning [25] to iteratively build an estimate of the State-Value function (22) and Q-Value function (23). For the State-Value function, the parameters $\Psi$ are thus optimized to minimize the TD-error:

$$
\begin{aligned}
J_{V}(\Psi)=V_{\Psi}^{\pi_{\mu}}\left(s_{t}\right)- & \left(\min \left[Q_{\Upsilon_{1}}^{\pi_{\mu}}\left(s_{t}, a_{t}\right), Q_{\Upsilon_{2}}^{\pi_{\mu}}\left(s_{t}, a_{t}\right)\right]\right. \\
& \left.-\log \pi_{\mu}\left(. \mid s_{t}\right)\right) .
\end{aligned}
$$

Similarly, the parameters $\Upsilon_{i}$ of the i-th Q-Value function estimator are optimized to minimize the TD-error:

$$
J_{Q}\left(\Upsilon_{i}\right)=Q_{\Upsilon_{i}}^{\pi_{\mu}}\left(s_{t}, a_{t}\right)-\left(r\left(s_{t}, a_{t}\right)+\gamma \times V_{\Psi^{\prime}}^{\pi_{\mu}}\left(s_{t+1}\right)\right),
$$

where $\gamma=0.99$ is the discount actor and $\Psi^{\prime}$ is defined in Section III-B2, The parameters $\mu$ of the Policy network are then optimized [18] in order to minimize the expected Kullback-Leibler (KL) divergence between the current policy and the exponential of the Q-Value function that is normalized by a function $Z_{\Upsilon}$ as:

$$
J_{\pi}(\mu)=\mathbb{E}_{s_{t} \sim D}\left[D_{K L}\left(\pi_{\mu}\left(\cdot \mid s_{t}\right)|| \frac{Q^{*}\left(s_{t}, \cdot\right)}{Z_{\Upsilon}\left(s_{t}\right)}\right)\right]
$$

where,

$$
Q^{*}\left(s_{t}, a_{t}\right)=\exp \left(\min \left[Q_{\Upsilon_{1}}^{\pi_{\mu}}\left(s_{t}, a_{t}\right), Q_{\Upsilon_{2}}^{\pi_{\mu}}\left(s_{t}, a_{t}\right)\right]\right), \forall s_{t}, a_{t} \text {. }
$$

When using the distribution expressed in Eq. (25) as a target for the policy shown in Eq. 24, the agent is forced to explore actions proportionally to their associated exponential Q-Values. This positive transformation allows a smarter exploration-exploitation tradeoff as negative Q-Values will be transformed into small but positive ones, forcing the policy to make progress along sub-optimal strategies until the algorithm finds which value is better for the future gains.

The gradient of $\nabla_{\mu} J_{\pi}(\mu)$ is approximated in [18] by:

$$
\begin{aligned}
& \hat{\nabla}_{\mu} J_{\pi}(\mu)=\nabla_{\mu} \log \pi_{\mu}\left(a_{t} \mid s_{t}\right)+\left(\nabla_{a_{t}} \log \pi_{\mu}\left(a_{t} \mid s_{t}\right)\right. \\
& \left.-\nabla_{a_{t}} \min \left(Q_{\Upsilon_{1}}^{\pi_{\mu}}\left(s_{t}, a_{t}\right), Q_{\Upsilon_{2}}^{\pi_{\mu}}\left(s_{t}, a_{t}\right)\right) \nabla_{\mu} f_{\mu}\left(\epsilon_{t}, s_{t}\right)\right) .
\end{aligned}
$$

The derivative in Eq. 26) is easier to compute compared to that in Eq. 24) and allow the use of Gradient Descent to optimize the parameters $\mu$ of the Policy ANN. Considering Eq. 21), the parameters $\mu$ are consequently optimized exactly for the desired maximum entropy objective (19). The soft Qupdate (23) guarantees that $Q^{\pi_{\text {new }}}\left(s_{t}, a_{t}\right) \geq \bar{Q}^{\pi_{\text {old }}}\left(s_{t}, a_{t}\right)$ and the repeated policy updates 26 ensure convergence toward the optimal policy $\pi^{*} 3$

\footnotetext{
${ }^{3}$ See Appendix B.2 and B.3 of $[18$ for the mathematical proof).
}

2) Stabilizing TD-Learning: The TD learning algorithm, summarized in Eq. 22] and 23], is different from Gradient Descent in the sense that the target value changes at each update, transforming the loss landscape. This generates training behaviors that are unstable and divergent. To tackle this problem, it is common practice to have a separate copy of the considered value network, denoted as "target" network, whose parameters are moving slowly or are fixed over a predefined amount of iteration steps. Here, we define a Target State-Value network $V_{\Psi^{\prime}}(s)$ that is then used to compute the Q-Value TD error (Eq. (23)) and thus with $\Psi^{\prime}$ slowly tracking the value of $\Psi$. In our case, we used the soft updates procedure which, for the target network parameters, consists in slowly tracking the parameter of the State-Value function using an exponential moving average $\Delta=0.005$. We used the Smooth L1 loss function from Pytorch [26] for the Critics optimization as it is less sensitive to outliers compared to standard MSE.

3) Artificial neural networks: Our implementation of the SAC algorithm is composed of 5 fully-connected Multilayer Perceptron (MLP): two Q-Value networks (with shared architectures), a Value and a Target-Value networks (with shared architectures) and a Policy network. We used the same ANN architecture as proposed in the original SAC paper [18] where each network is composed of 2 hidden layers of 256 hidden units each. The Pytorch framework [26] and CUDA toolkit [27] were used to implement this architecture along with an Nvidia RTX 2070 GPU card for the gradient and simulation processing. The SAC algorithm builds a stochastic policy where the action distributions are modeled by Gaussian distributions 4 There are several advantages of considering a stochastic policy: it prevents early convergence of the policy variance, it encourages exploration in the value function by increasing the value of regions of state space that lead to highentropy policy, and the resulting policy tends to perform much more consistently compared to its deterministic counterpart with improved robustness to uncertainties [18]. We used Adam [28] as optimizer for all networks with the learning rate $l_{r}=3 e^{-4}$. The Leaky ReLU activation function is applied to all hidden layers and gradient descent is applied using a mini-batch of size 256. Layer Normalization [29] (LN) is added before the activation function of all the hidden layers and L2 weight penalty of $1 e^{-3}$ is incorporated to the Critics only. The weights and biases are initialized from the Gaussian distribution $\mathcal{N}(0, \sqrt{2 / f})$, where $f$ is the fan-in of the layer.

\section{A Bio-INSPIRED EXPERIENCE REPLAY}

This section presents the ER method proposed in this paper, which is called BIER for Bio-Inspired ER. In this method, the agent experience is divided into two distinct memory units (as illustrated in Figure 1) and samples are drawn from them differently as described next.

Sequential-Partial Memory: this buffer is denoted as $B_{1}$ and is similar to the one from the original ER scheme. Here its maximum size is set to $1,000,000$, and contains old and new transitions. We believe that, especially in the robotic case,

\footnotetext{
${ }^{4}$ See appendix $\mathrm{C}$ of $[18$ for more details on the change of variable applied to bound the Gaussian distributions
} 
the optimal behavior is highly temporally correlated. With robots, early actions do have an impact on the future states. Learning this temporal relationship is thus essential in order to learn what a truly good behavior, in the physical sense, is. In addition, even with very different operating conditions, the robot's behavior remains quite similar. This means that the shape of the trajectories remains within a bounded space. Therefore, our hypothesis is that learning on sequences can lead to further gains (compared to temporally uncorrelated samples) because what we learn on a trajectory can directly be applied to future ones that the agent has yet to encounter. Following this intuition, we propose to sample random sequences of transition from Buffer B1 (i.e. transitions that are successive within the buffer since we store each of them in a first-in-firstout fashion). The vector of experiences $E$ sampled from $B_{1}$ is composed of $n$ samples as $E=\left[e_{i} ; e_{i+1} ; \ldots ; e_{i+n}\right]$, for $0<i<m-n$.

While this replay procedure sounds very appropriate for a biological system, here we are optimizing MLPs with limited numbers of parameters and learning abilities. Using highly correlated samples when performing gradient descent often leads to overfitting or local minima. In fact, sequential states are strongly correlated. This is often observed in onpolicy methods where the ANNs are optimized using samples generated by the current policy. This usually leads to repeatedly overfitting to those local correlated samples and never really learning the true value of the functions (i.e. it ends up oscillating between different overfitting regimes). For this reason, we propose to consider sequences that are only partial by storing 1 out of 2 transitions in the buffer $B_{1}$ which:

- adds a regularization effect by feeding incomplete sequences to the ANNs that encourages these networks to further learn the real value of the functions.

- reduces the age of the oldest policy contained in this buffer, which improved performance in [12].

Optimistic Memory: both bad and good behaviors are important when learning a new task because they both contain information about the process. We have been able to observe a number of cases where "positive reinforcing" is much more efficient with biological systems, for example with animal training where good and bad behaviors are respectively rewarded with treats or no response (rather than punishment). It was shown in [12] that trying to estimate values of highquality regions (as measured by the rewards) results in better performance. In addition, as the agent learns, its performance improves and better transitions are performed. Such transitions are important because they can further improve the data collection of the agent in the future. However, as shown in [10], when using a large replay buffer, such transitions are likely to influence the policy later. Their probability to be sampled decreases as the replay buffer size increases, slowing down performance improvement. Following these heuristics, our objective with this second buffer $B_{2}$ is to be optimistic about past experience, by increasing the probability of using transitions associated with such high-quality regions.

We propose to store in $B_{2}$ the upper outliers of the reward distribution that we consider to be the best transitions. Outliers can be defined according to diverse metrics depending on the nature of the variable distribution. The challenge is that we can not predict the distribution shape beforehand. For instance, with our reward function, the closer the robot gets to the setpoint, the higher the maximum value of possible reward becomes (hence, the optimal policy should lead to a reward distribution of Pearson shape). In practice, however, the closer the vehicle is to the setpoint the more difficult it becomes for it to physically reduce the errors (which is more akin to a Gaussian distribution). Depending on the system, the operating conditions, and the reward function (among others), the reward distribution can switch between various shapes, potentially making the predefined metric not robust to different distribution assumptions. Thus, we propose to consider a transition as an outlier of interest and to store it in $B_{2}$ if its associated reward $r\left(s_{t}\right)$ is:

$$
r\left(s_{t}\right)>\mathbb{E}\left[r\left(s_{t}\right)\right],
$$

where the expected value $\mathbb{E}\left[r\left(s_{t}\right)\right]$ is computed over the last 50,000 rewards generated that are stored as an additional variable $M$. The size of $M$ was chosen in order to compute the expected reward over a moving window of approximately 100 episodes in order to give more importance to novel inputs, similarly to biological systems [13].

This choice of expected value as a metric is related to the subtracted baseline in Eq. (26) that is the Advantage function $A(s, a)$. This function represents the benefits of changing the current policy. If the value of $A(s, a)$ is positive, then the probability to take the evaluated action (with respect to. the given state) will be increased because its Q-Value is higher than the expected one (with respect to. the current policy). Our assumption is that transitions that meet the criteria expressed in Eq. (27) are associated with positive values of $A(s)$. Gradient updates using samples from the Optimistic Memory will therefore mostly improve the expected return of the policy, leading to faster discovery of successful trajectories.

The maximum size of $B_{2}$ is set to 10,000 . It is drastically smaller than $B 1$ because, as the agent's performance improves over the course of training, what was considered as a good transition is most likely to be outdated. Therefore, the reduced buffer size ensures that we focus on the current best transitions. Finally, contrary to the first buffer, we propose to sample $n$ uncorrelated items from $B_{2}$ as single transitions are iteratively stored in this buffer.

\section{TRAINING}

This section describes the training settings (including training scenario, reward function, state vector, and exploration strategy) with the hyperparameters choice. It ends with an analysis of the training results.

\section{A. Scenarios definition}

The training consists of performing a total of 3000 episodes (as no further notable improvement in setpoint regulation is observed after approximately 2500 episodes). The maximum length of a training episode was set at 500 timesteps (equivalent to 25 seconds). We define a training episode as follows: 


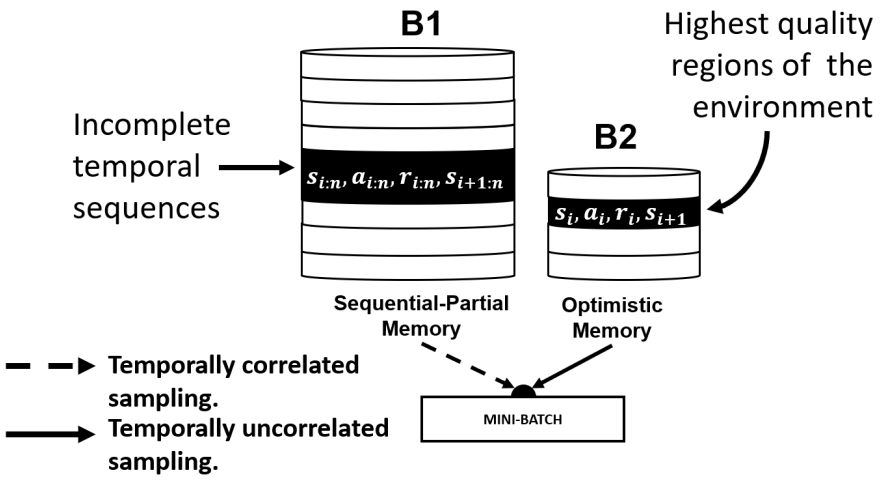

Fig. 1. Illustration of our bio-inspired procedure. The agent's past experience is divided in two memory units: 1 out of 2 transitions are stored in the first buffer and we sample from it temporal sequences of experiences; we store in the second buffer the best transitions as measured by reward and with respect to the current policy. This procedure takes advantage of the resilience from on-policy sampling while keeping the data efficiency from the off-policy formulation.

1) The robot is initialized at a depth of 40 meters with a random orientation $(\psi, \theta, \phi) \in\left[\frac{-\pi}{4} ; \frac{\pi}{4}\right]$ and at null velocity.

2) The current variables are randomly chosen such that $v_{c} \in$ ] $0,0.5]$ and $\left[h_{c}, j_{c}\right] \in\left[\frac{-\pi}{4} ; \frac{\pi}{4}\right]$ (which remain fixed during the episode) and a random vector of setpoints is generated such that $x_{r e f}=\left[v_{x}, 0,0,0,0,0\right]^{T}$ with $\left.v_{x} \in[0.1,0.5]\left(m . s^{-1}\right) .3\right)$ Then, the off-policy $\pi_{\mu}(a \mid s)$ behavior is used.

4) The episode ends when the control objective (2) is met or when the episodic step number exceed 500 .

\section{B. Reward shaping}

Compared to our previous work [30], the control objective treated here is fundamentally more complex since we are not trying to reach one singular state but rather a sequence of desired successive states. We propose the following terminal reward signal to take this into account:

$$
r_{\text {success }}=1000 \text { if } \forall t \in[t-100, t],\left|e_{i}(t)\right| \leq \chi .
$$

Therefore, performing the desired control objective, as shown in Eq. (2), will generate this reward, Eq. (28), and its value was chosen in order to make sure that, for all trajectory lengths, the maximum sum of return is obtained only by stabilizing the vehicle. Otherwise, the reward $r\left(s_{t}\right)$ is generated. Let's define the Euclidean distance to the desired setpoint as $e_{L 2}(t)=\sqrt{\sum_{i=1}^{i=\operatorname{dim}(u)} e_{i}^{2}(t)}$ and its derivative is computed over the last two frames and denoted as $d_{\text {rate }}(t)$. The reward $r\left(s_{t}\right)$ is then defined as:

$$
r\left(s_{t}\right)=C_{1} \times \exp \left[-e_{L 2}^{2}(t) \times C_{2}\right]
$$

The performance of SAC is highly dependent on the choice of reward scale (or amplitude) which, in the case of our reward function Eq. (29), is controlled by the constant $C_{1}$. The reward scale can be interpreted as the inverse of the temperature parameter $\alpha$ from 19$]$ which controls the stochasticity of the resulting policy. Here, we empirically chose $C_{1}=40$, which gave us the best performance, by following advice from [20]. The reward signal, Eq. 29, is equal to its maximum value possible per step (that is $C_{1}$ ) only when all the current errors are equal to zero. As AUVs move slowly, successive states display error signals $e_{i}(t)$ of minor and similar amplitude. We find that this addition of $C_{2}=10$ (compared to our previous work [15]), makes it easier for the critics to differentiate the State-Value of successive states without altering the reward scale as $\lim _{x \rightarrow 0} C \times e^{x}=C$. This reward function, Eq. [29], encourages the agent to reduce the errors as much and as fast as possible and the vehicle stabilization is further promoted by generating the maximum reward possible per step, as shown in Eq. (28). In order to improve the exploration ability provided by the maximum entropy framework, we used adaptive parameter noise [31] which consists in adding noise to the parameters of the policy network.

\section{Process Observability}

At each timestep, the agent captures an observation vector $o_{t}$ representing the process dynamics that we defined as:

$$
o_{t}=\left[a_{t-1} ; \Theta ; V ; \Omega ; u_{t} ; e_{t} ; e_{L 2} ; d_{\text {rate }} ; \delta_{\chi}\right],
$$

where $a_{t-1} \in \mathbb{R}^{18}$ are the last action estimated (i.e. poles value); $\Theta=[\phi ; \theta ; \psi]$ are the Euler orientation of the vehicle (roll, pitch and yaw respectively); $V=\left[v_{x} ; v_{y} ; v_{z}\right]$ and $\Omega=\left[\omega_{\phi} ; \omega_{\theta} ; \omega_{\psi}\right]$ are its linear and angular velocities; $u_{t} \in \mathbb{R}^{6}$ are the last control inputs applied; $e_{t} \in \mathbb{R}^{6}$ are the error values on each setpoint; $e_{L 2}$ and $d_{\text {rate }}$ as described in Section $\mathrm{V}$-B and $\delta_{\chi} \in[0,1]$ is a variable which keeps track of the number of successive steps where all the errors are within the threshold (i.e. if $\delta_{\chi}=1$, the control objective is achieved). The dimension of the observation vector $o_{t}$ is therefore equal to 42 . Noted that with this observation vector (30), the current disturbance characteristics are not included. In order to improve the process observability and following our previous results [32], we construct our state vector $s_{t}$ out of the current and past observation vectors along with their two-by-two difference. This results in a 126 dimensional state space defined as $s_{t}=\left[o_{t} ; o_{t-1} ; o_{t-1}-o_{t}\right]$.

\section{Training results}

Figure 2 shows the training dynamics with the normalized mean return per episode, the RMSE on the setpoint per episode, and the normalized mean return standard deviation per episode. The yellow dashed lines represent the performance of the OFP controller. It is fair to compare these controllers, as they are based on the exact same control structure as our learning-based controller, that is the PID structure. Thus, the only difference between the three controllers is the value of the poles used to compute the PID control inputs.

As we can see in Figure 2, both methods are able to learn the task and converge toward what seems to be a maximum value of the reward. In the third plot of Figure 2, we can see that the performance improvement is smoother with the BIER method which exhibits a lower reward standard deviation (which tends to reduce over time contrary to the CER agent). With the CER method, the variance is higher, with pikes that even drive the agent to lower performance than those obtained with the OFP controller. Noted that the OFP controller displays the lowest 
standard deviation (third plot of Figure 2), thanks to the model information incorporated in SMAC [17].

The agent trained using the BIER method was able to exceed the performance of the OFP controller, in less than half the number of episodes compared to the standard CER method. It is represented in Figure 2 by the vertical lines. Therefore, when DRL can be considered on the physical platform, a designer might favor the use of our BIER method for improved data efficiency and learning stability.
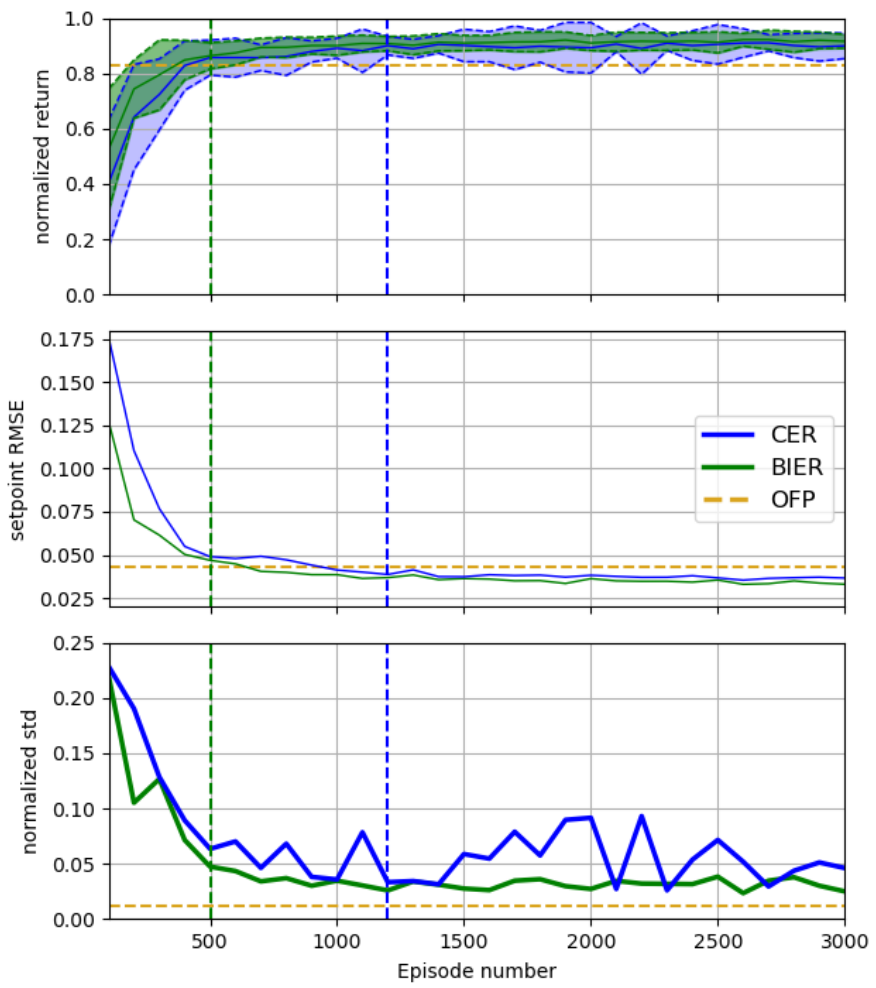

Fig. 2. Training curves for both Experience Replay methods. The BIER agent outperforms the CER agent in both learning speed and variance. Both learning-based controllers outperform the model-based controller (i.e. OFP).

\section{EXPERIMENTS AND ANALYSIS}

The challenge of conducting DRL in real-life lies in the drop in performance caused by the distribution shift that is here the difference between the training and evaluation set of states. In order to visualize this behavior in our use case, we present below different evaluation scenarios of increasing complexity as measured by desired setpoint and current velocity.

Scenario 1: the setpoint range is the same as during training but no current disturbances is applied. This scenario is therefore in theory simpler than the training one.

Scenario 2: the process is the same as during training but with setpoint and current variables that were not seen during training (but are still in the same range).

Scenario 3: the setpoint $v_{x}$ is increased to the range $[0.5,1.0]$, the current variables are the same as during training.

Scenario 4: the setpoint characteristic is the same as during training but the current velocity $v_{c}$ is increased to $[0.5,1.0]$ $\left(m . s^{-1}\right)$ and $\left(h_{c}, j_{c}\right) \in\left[-\pi, \frac{-\pi}{2}[\cup] \frac{\pi}{2}, \pi\right]$.
Scenario 5: both the setpoint and current variables are increased to the range defined in scenarios 3 and 4 .

Scenario 6: we keep the increased range from scenario 5 , and at a random timestep during the episodes (between the 100th and 400th timestep), we vary the current variables (velocity and orientation) within the same training range.

In Table I] the metrics were computed over 500 episodes (different from each other) for each evaluation scenario. The line "Baseline" denotes the performance obtained at the end of the training, which incorporates the parameter noise described in Section V-B. For the other scenarios, this noise is removed. In order to visualize more easily the performance variation, we propose in Figure 3 an illustration of these results.

The CER and BIER agents are able to stabilize the vehicle over the first 3 scenarios where the performance is matching the training one. When increasing the desired setpoint value, we can see the associated RMSE slightly increasing but the performance remains satisfying. The performance drop is the largest when increasing the sea current disturbance (i.e. scenarios 4,5 , and 6). The sensibility to this disturbance is further depicted by the performance difference that is much smaller between scenarios 5 and 6 compare to between scenarios 3 and 4 . We believe this is due to the current characteristics not being explicitly included in the state vector.

The BIER agent performs better on scenario 1 compared to scenario 2 which is simpler than the training scenario as it does not incorporate sea current disturbance. More interestingly, its performance compared to CER has also increased on scenarios 5 and 6 , despite the distribution shift being particularly large there. This suggests that the policy obtained with the BIER method has better generalization abilities. We can see that the OFP controller is much sensitive to sea current disturbance compare to setpoint variation (as it is included in the model). Noted that the learning-based controllers are able to exceed the OFP controller on scenario 6, despite not having experience such disturbance during training.

These results show that ER does have an impact on the quality of the resulting policy. By only manipulating differently the agent's past experience, we were able to make learning faster, and with improved robustness to increased process variations. Reducing the distribution shift problem in DRL remains an active field of research and future work could focus i) on improving process observability and ii) studying how conservative can a DRL-based policy be.

\section{CONCLUSION}

In this article, we proposed a new Bio-Inspired Experience Replay which aims at incorporating concepts from the biological Replay Mechanism in the context of DRL. We found that by taking inspiration from nature, while keeping in mind the requirements from Gradient-based optimization, the adaptive controller can learn faster and with improved stability. Our results also suggest that learning-based adaptive control might be a key ingredient toward real-life autonomous robotic applications, by taking advantage of the available information on the process, with a model-based structure, and using modelfree learning to adjust its parameters to compensate for unmodeled process variation, via Deep Reinforcement Learning. 
TABLE I

EVALUATION RESULTS FOR THE CER METHOD (LEFT) AND THE PROPOSED BIER METHOD (RIGHT).

\begin{tabular}{|c|c|c||c|c|}
\hline Scenario & mean RMSE per step & normalized mean return & mean RMSE per step & normalized mean return \\
\hline Baseline & 0.0364 & $0.9104 \pm 0.0461$ & $\mathbf{0 . 0 3 3 0}$ & $\mathbf{0 . 9 2 1 9} \pm \mathbf{0 . 0 2 5 0}$ \\
1 & 0.0370 & $0.9072 \pm 0.0309$ & $\mathbf{0 . 0 3 6 6}$ & $\mathbf{0 . 9 3 4 7} \pm \mathbf{0 . 0 2 6 2}$ \\
2 & 0.0350 & $0.9108 \pm 0.0456$ & $\mathbf{0 . 0 3 2 0}$ & $\mathbf{0 . 9 2 4 4} \pm \mathbf{0 . 0 2 4 0}$ \\
3 & 0.0448 & $0.8774 \pm 0.0416$ & $\mathbf{0 . 0 4 1 8}$ & $\mathbf{0 . 9 1 2 4} \pm \mathbf{0 . 0 2 6 6}$ \\
4 & 0.1483 & $0.4078 \pm 0.2965$ & $\mathbf{0 . 1 2 1 4}$ & $\mathbf{0 . 5 0 7 1} \pm \mathbf{0 . 2 5 3 0}$ \\
5 & 0.1656 & $0.3556 \pm 0.2846$ & $\mathbf{0 . 1 5 0 8}$ & $\mathbf{0 . 4 2 8 9} \pm \mathbf{0 . 2 5 7 3}$ \\
6 & 0.1802 & $0.3238 \pm 0.2219$ & $\mathbf{0 . 1 6 3 7}$ & $\mathbf{0 . 3 9 6 6} \pm \mathbf{0 . 2 1 6 7}$ \\
\hline
\end{tabular}

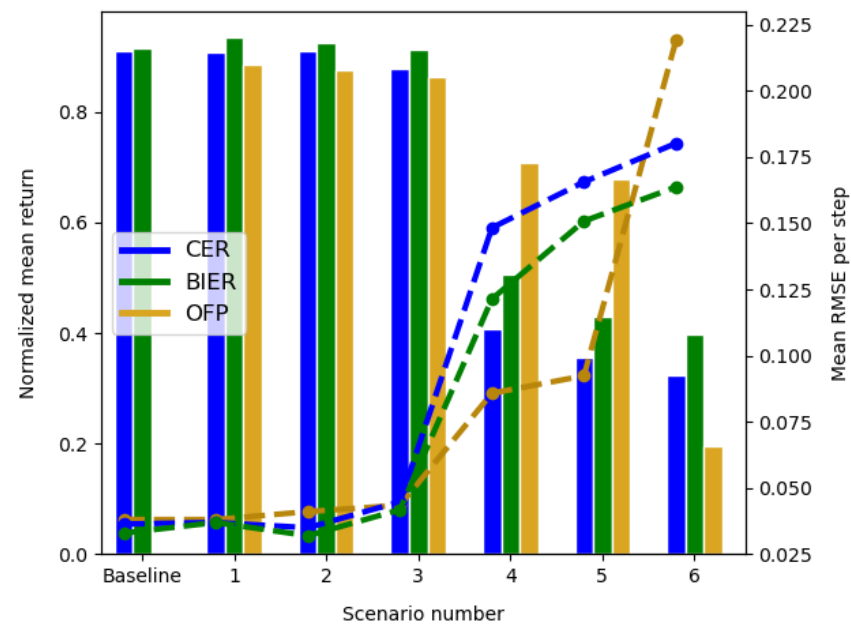

Fig. 3. Illustration of the evaluation performance. The BIER agent performs consistently better than the CER one despite having been trained using a reduced variety of samples.

\section{REFERENCES}

[1] K. Astrom and B. Wittenmark, Adaptive Control. Dover Publications, 2008.

[2] R. S. Sutton and A. G. Barto, Reinforcement learning an introduction Second edition. MIT Press, 2018.

[3] S. Padakandla, "A survey of reinforcement learning algorithms for dynamically varying environments," ACM Comput. Surv., vol. 54, no. 6, 2021.

[4] V. Konda and J. Tsitsiklis, "Actor-critic algorithms," Advances in neural information processing systems, vol. 12, 1999.

[5] R. Yu, Z. Shi, C. Huang, T. Li, and Q. Ma, "Deep reinforcement learning based optimal trajectory tracking control of autonomous underwater vehicle," 2017 36th Chinese Control Conference (CCC), 2017.

[6] C. Wang, L. Wei, Z. Wang, M. je Song, and N. Mahmoudian, "Reinforcement learning-based adaptive trajectory planning for auvs in underice environments," OCEANS 2018 MTS/IEEE Charleston, 2018.

[7] K. B. Knudsen, M. C. Nielsen, and I. Schjølberg, "Deep learning for station keeping of auvs," OCEANS 2019 MTS/IEEE Seattle, 2019.

[8] Z. Chu, B. Sun, D. Zhu, M. Zhang, and C. Luo, "Motion control of unmanned underwater vehicles via deep imitation reinforcement learning algorithm," Iet Intelligent Transport Systems, vol. 14, pp. 764-774, 2020.

[9] L. Lin, "Self-improving reactive agents based on reinforcement learning, planning and teaching," Machine Learning, vol. 8, pp. 293-321, 2004.

[10] S. Zhang and R. Sutton, "A deeper look at experience replay," arXiv preprint arXiv:1712.01275, 2017.

[11] T. Schaul, J. Quan, I. Antonoglou, and D. Silver, "Prioritized experience replay," CoRR, vol. abs/1511.05952, 2016.

[12] W. Fedus, P. Ramachandran, R. Agarwal, Y. Bengio, H. Larochelle, M. Rowland, and W. Dabney, "Revisiting fundamentals of experience replay," in International Conference on Machine Learning, 2020.

[13] T. L. Hayes, G. P. Krishnan, M. Bazhenov, H. T. Siegelmann, T. J. Sejnowski, and C. Kanan, "Replay in deep learning: Current approaches and missing biological elements," Neural Computation, vol. 33, no. 11, pp. 2908-2950, 2021
[14] M. Manhães, S. Scherer, M. Voss, L. Douat, and T. Rauschenbach, "UUV simulator: A gazebo-based package for underwater intervention and multi-robot simulation," in MTS/IEEE OCEANS, Monterey, 2016.

[15] T. Chaffre, G. Le Chenadec, K. Sammut, E. Chauveau, and B. Clement, "Direct adaptive pole-placement controller using deep reinforcement learning: Application to auv control," IFAC-PapersOnLine, vol. 54, no. 16, pp. 333-340, 2021, 13th IFAC Conference on Control Applications in Marine Systems, Robotics, and Vehicles CAMS.

[16] A. O'Dwyer, Handbook of PI and PID controller tuning rules. Imperial College Press, 2006.

[17] F. Hutter, H. Hoos, and K. Leyton-Brown, "Sequential model-based optimization for general algorithm configuration," in LION, 2011.

[18] T. Haarnoja, A. Zhou, P. Abbeel, and S. Levine, "Soft actor-critic: Offpolicy maximum entropy deep reinforcement learning with a stochastic actor," in Proceedings of the 35th International Conference on Machine Learning, 2018

[19] T. Haarnoja, A. Zhou, K. Hartikainen, G. Tucker, S. Ha, J. Tan, V. Kumar, H. Zhu, A. Gupta, P. Abbeel, and S. Levine, "Soft actorcritic algorithms and applications," CoRR, vol. abs/1812.05905, 2018.

[20] T. Haarnoja, S. Ha, A. Zhou, J. Tan, G. Tucker, and S. Levine, "Learning to walk via deep reinforcement learning," arXiv preprint arXiv:1812.11103, 2019.

[21] T. Haarnoja, H. Tang, P. Abbeel, and S. Levine, "Reinforcement learning with deep energy-based policies," in Proceedings of the 34th International Conference on Machine Learning, ICML. JMLR.org, 2017, pp. 1352-1361.

[22] Z. Ahmed, N. L. Roux, M. Norouzi, and D. Schuurmans, "Understanding the impact of entropy on policy optimization," in ICML, 2019.

[23] H. V. Hasselt, A. Guez, and D. Silver, "Deep reinforcement learning with double q-learning," in Proceedings of the AAAI conference on artificial intelligence, 2016.

[24] S. Fujimoto, H. Van Hoof, and D. Meger, "Addressing function approximation error in actor-critic methods," in Proceedings of the 35th International Conference on Machine Learning, ICML, 2018.

[25] R. S. Sutton, "Learning to predict by the methods of temporal differences," Machine Learning, vol. 3, pp. 9-44, 2005.

[26] A. Paszke, S. Gross, F. Massa, A. Lerer, J. Bradbury, G. Chanan, T. Killeen, Z. Lin, N. Gimelshein, L. Antiga, A. Desmaison, A. Köpf, E. Yang, Z. DeVito, M. Raison, A. Tejani, S. Chilamkurthy, B. Steiner, L. Fang, J. Bai, and S. Chintala, "Pytorch: An imperative style, highperformance deep learning library," in NeurIPS, 2019.

[27] J. Nickolls, I. Buck, M. Garland, and K. Skadron, "Scalable parallel programming with CUDA," EEE Hot Chips 20 Symposium (HCS), 2008.

[28] D. P. Kingma and J. Ba, "Adam: A method for stochastic optimization," in 3rd International Conference on Learning Representations, ICLR, Y. Bengio and Y. LeCun, Eds., 2015.

[29] L. J. Ba, J. R. Kiros, and G. E. Hinton, "Layer normalization," CoRR, vol. abs/1607.06450, 2016.

[30] T. Chaffre, J. Moras, A. Chan-Hon-Tong, J. Marzat, K. Sammut, G. Le Chenadec, and B. Clement, "Learning-based vs model-free adaptive control of a mav under wind gust," in Informatics in Control, Automation and Robotics. Springer, 2022, pp. 362-385.

[31] M. Plappert, R. Houthooft, P. Dhariwal, S. Sidor, R. Y. Chen, X. Chen, T. Asfour, P. Abbeel, and M. Andrychowicz, "Parameter space noise for exploration," in 6th International Conference on Learning Representations, ICLR, 2018.

[32] T. Chaffre, J. Moras, A. Chan-Hon-Tong, and J. Marzat, "Sim-to-real transfer with incremental environment complexity for reinforcement learning of depth-based robot navigation," in 17th International Conference on Informatics, Automation and Robotics, ICINCO, 2020. 